\title{
ANN based Short-Term Load Curve Forecasting
}

\author{
V. Chis, C.Barbulescu, S. Kilyeni, S. Dzitac
}

\author{
Violeta Chis \\ Mathematics and Computer Science Department \\ Aurel Vlaicu University of Arad \\ Arad, Romania \\ violeta.chis@uav.ro \\ Constantin Barbulescu*, Stefan Kilyeni \\ Power Systems Department \\ Politehnica University Timisoara Romania \\ Timisoara, Romania \\ *Corresponding author: constantin.barbulescu@upt.ro \\ stefan.kilyeni@upt.ro \\ Simona Dzitac \\ Power Engineering Department \\ University of Oradea \\ Oradea, Romania \\ simona@dzitac.ro
}

\begin{abstract}
A software tool developed in Matlab for short-term load forecasting (STLF) is presented. Different forecasting methods such as artificial neural networks, multiple linear regression, curve fitting have been integrated into a stand-alone application with a graphical user interface. Real power consumption data have been used. They have been provided by the branches of the distribution system operator from the Southern-Western part of the Romanian Power System. This paper is an extended variant of $[4]^{a}$.

Keywords: artificial neural networks; short-term load forecasting; artificial intelligence; load curve.

\footnotetext{
${ }^{a}$ Partially reprinted and extended, with permission based on Licence Number 4453460839057 C IEEE, from "2018 7th International Conference on Computers Communications and Control (ICCCC)."
}

\section{Introduction}

The current paper is an extended version of [4]. It deals with real power systems data applied for short-term daily load forecasting. In [4] one single distribution branch has been discussed. Currently, the work has been extended for all of the distribution branches belonging to the distribution system operator (DSO) involved. A new set of data, corresponding to the entire network of the DSO has been added. Finally, a comparative analysis has been provided for all the distribution branches involved and the entire network assembly.

Load forecasting has a great impact on future decisions, predicting the energy demand for the operation and planning of power systems plays an important role in the control, power security, market operation, and scheduling of reasonable dispatching plans for smart grids.

Load forecasting is an important component of power system to establish economical and reliable operations for power stations and their generating units. An accurate load forecasting approach used to predict load demand is essential part of any energy management system.

The load forecasting methods are generally classified in statistical-based methods like exponential smoothing, regression, Kalman filter, state space model and artificial intelligence based 
methods such as expert system techniques [7], neural networks (NN), fuzzy expert system [20], fuzzy time series, fuzzy neural networks, genetic algorithms [8], support vector machine, pattern recognition and hybrid methods.

Load forecasting is carried out over a wide range of intervals ranging from a few seconds to decades and is mainly based on retrospective load variation data. According to the time span, load forecasting can be divided into very short (a few minutes), short term load forecasting (STLF), medium term load forecasting (MTLF) and long term load forecasting (LTLF).

LTLF covers a period of several years and is applicable for system and long term network planning. The MTLF covers a period of a few weeks and is applicable for scheduling of the fuel supply, the planning of maintenance, etc. The STLF refer to hourly prediction of the load for a lead time ranging from one hour to several days out and it's used to predict load demands so that the day-to-day operation of a power system can be efficiently planned [9].

The accuracy of load forecasting depends significantly on the availability of historical consumption data as well as on the knowledge about the main influence parameters on the energy consumption [16].

The quality of the forecast methods is influenced by several factors including:

- weather data- temperature, humidity, wind speed, fog, precipitation, solar radiation;

- seasonal (time) factors - seasons' sequence, day of the week and hour of the day (with low periods during the night hours and with peaks at different day hours);

- causative factors (holidays, strikes and some specific public events, etc.);

- economic factors (industrial activity, demographic change, economic growth).

The influence of these factors is different from one type of forecast to another.

Short-term load forecasting (STLF) performs load forecasting of the system from few hours to weeks, using generally historical load data and weather data as inputs. The forecasted data are used to estimate load flows, for transmission line loading, for transient stability studies. Many decisions, like generating capacity, planning for energy transactions or system security assessment are based on STLF.

Researchers have considered different approaches to STLF, like time series method, regression analysis [1], intelligent techniques, such as NN [2], [17], fuzzy logic [10], [14], neuro-fuzzy [6] or data mining [18], [21].

Time series have been used for decades in areas such as the economy, digital signal processing and the load forecasting. ARMA (AutoRegressive Moving Average) [3], ARIMA (Auto Regressive Integrated Moving Average) [11] and ARIMAX (AutoRegressive Moving Average with Exogeneous Variables) [5] are the most commonly used time series methods.

Since 1990, the focus has been on using different Artificial Intelligence (IA) techniques. Thus, the authors of the paper [15] were among the first research groups that chose to use NN for the STLF. In [12], a model based on decision trees is used for load forecasting.

In [19] the neural networks are also used for load forecasting problem, due to the non-linear character of the load. But, the authors are going further, combining the ANNs with optimization techniques. They are proving that the forecast error is reduced by applying this method, in order to estimate the parameters of the network.

Another hybrid model is applied in [22]. The authors are focusing on empirical mode decomposition (IEMD), autoregressive integrated moving average (ARIMA) and wavelet neural network (WNN). All these algorithms are optimized by fruit fly optimization algorithm (FOA). This kind of approach is recommended in case of load forecasting affected by natural and social factors. 
Paper [13] is one of the papers that are dealing with dynamic neural networks, in order to predict the daily power consumption. These networks are able to adaptively learn de patterns from historical data. They are capable to tackle the high non-linear degree between input and output data.

The introduction is presented within the 1st section of the paper. The 2nd one focuses on describing the applied models. The software-tool is briefly presented within the $3 \mathrm{rd}$ one. The case study and the results are largely discussed within the 4th section. Finally, the 5th one synthesizes the conclusions.

\section{Models used}

Several models have been implemented within the developed software tool.

\subsection{Multiple linear regression model}

Regression methods are used to model the relationship between load consumption and other factors such as weather, day type, stochastic influences such as average loads and customer class [23].

The following data have been used within the current model: previous day, type of the day (working day or weekend), previous day same hour load and previous 24 hour average load.

The following stages have been tackled:

- generate predictors (previousDayHour, pre24HourAverLoad, day, dayweek);

- forming validation input data (vpreviousdayHour, vpre24HourAverLoad, vday, dayweek);

- create regression coefficients $(b$, bint,$r)$;

- validate the regression coefficients (evaluating the input data) - calculate the mean absolute percent error (MAPE) and plot actual load vs. predicted load;

- use the regression coefficients to forecast one day ahead - calculate MAPE and plot actual load vs. forecasted load.

- use the regression coefficients to forecast one day ahead - calculate MAPE and plot actual load vs. forecasted load

The time factors include the time of the day of the week, and the day hour. There are important differences in load between weekdays and weekends. The load on different weekdays also can behave differently.

\subsection{Curve fitting model}

From the Fourier library models, fourier8 has been used. Multiple regression has been used to obtain an average estimate. Figure 1 presents synthetically the algorithm of this method for STLF. 


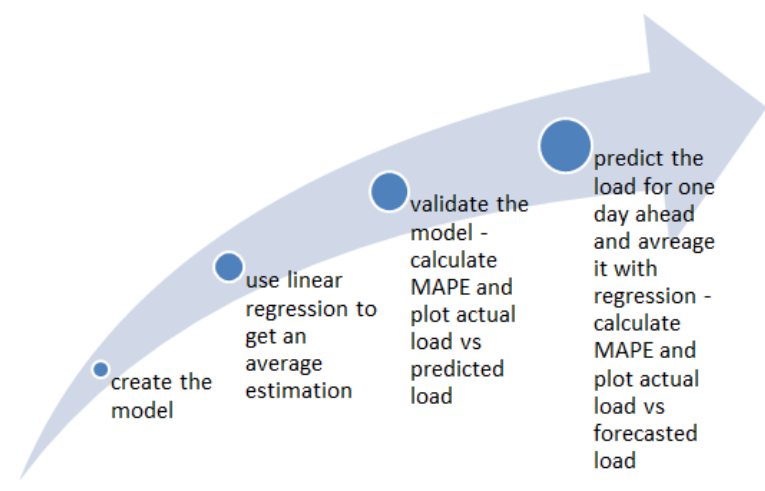

Figure 1: Curve fitting algorithm

\subsection{The neural network model}

Several papers propose the use of NN for complex problems like STLF, which are highly non-linear. The main advantages of $\mathrm{NN}$ are that it can learn to gain on any nonlinear function from a large number of data.

To identify the assumed relation between the future load and the earlier load data a multilayer perceptron (MLP) network with a single hidden layer is used. MLP is one of the most well-known $\mathrm{NN}$ architectures for prediction algorithms, and is popular because of its flexibility in assuming shapes of complex patterns. The introduction of hidden layer(s) makes it possible for the network to exhibit non-linear behavior. For calculation of hidden layer neurons does not exist any specific formula. Researchers have observed that too few hidden layer neurons can cause the network to not learn the convergence and too many hidden layers can cause the network to memorize the scheme rather than forecasting [2], [17].

The NN is trained using historical data and use back propagation (BP) method. BP is widely applied for STLF because of its ability to study and remember the relation between inputs and outputs as well as to approach any types of function. For training, the network uses the default BP training algorithm, Levenberg-Marquardt. This is the fastest method in the toolbox for training moderate-sized feed-forward neural networks. The overall design of NN is presented in Figure 2.

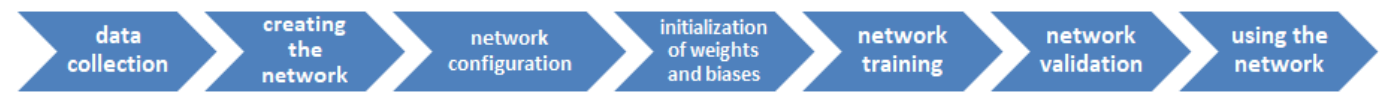

Figure 2: The overall design of NNs

For the current paper the NN architecture has the following configuration: one output (load), six inputs: load on previous day at same hour, load on previous week at same hour, month, day, day of the week (there are important differences in load between weekdays and weekends).

\section{Software tool}

A software tool has been developed in Matlab environment for STLF. Of course, there are commercial software packages. Their main disadvantage is that they are a black box, offering no modeling transparency and they are more difficult to modify.

Data are retrieved from Microsoft Excel files. For different loads, the influence of different factors is not the same: temperature could have a major influence for residential loads, but may 
have little impact on some industrial loads. In this case the temperature has not been taken into account. Future power demand is estimated based on the historical load data.

The developed software allows a quick graphical comparative analysis: Actual Load versus Load Forecasting. The MAPE is used as a performance criterion.

$$
M A P E=\frac{1}{N}\left(\sum_{i=1}^{n} \frac{\left|y_{i}-p_{i}\right|}{y_{i}}\right) * 100
$$

where $y_{i}, p_{i}-$ actual and forecasted load of $i$ hour; $N$ - the forecasting horizon

This application was developed using the Matlab GUI Tool-box and implements different models. The models developed have been integrated into a stand-alone application with a graphical user interface.

Starting from the idea that the interface design activity should be centered on the user, it was intended to meet the recommended requirements for such interfaces: friendly, intuitive, easy-touse, extensible but also consistent. Radio buttons have been used to select the model (Figure 3). Depending on data, one may be better than another. The developed software allows comparing different models automatically.

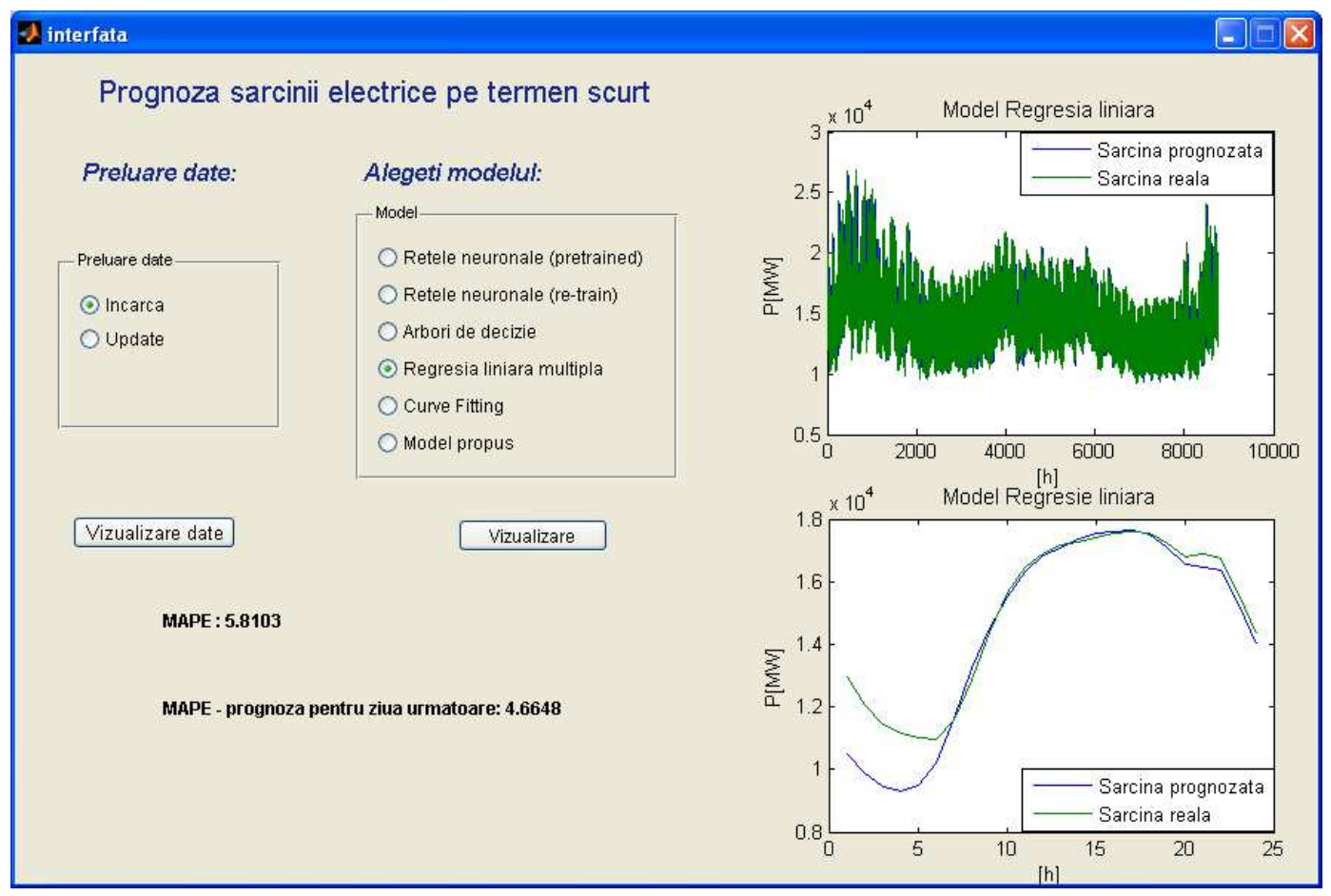

Figure 3: The software tool main window

\section{Results and discussions}

Real data have been used, that are belonging to the Distribution System Operator managing the distribution network from the Western side of Romania. The load curves forecasting is performed for the most significant summer day ( $21^{\text {st }}$ June). 10 years known period is considered (2004-2013). Results validation refers to the range of 2014-2016 years. The forecasts have been performed using artificial neural networks (ANN), multiple linear regression (MLR) and curve fitting $(\mathrm{CF})$. 
Table 1 and Figure 4 contain the known load curves for the 2004-2013 period, for the most significant summer day.

Table 1: Load curves for known period 2004-2013 [MW]

\begin{tabular}{|c|c|c|c|c|c|c|c|c|c|c|}
\hline Year/Hour & $\mathbf{2 0 0 4}$ & $\mathbf{2 0 0 5}$ & $\mathbf{2 0 0 6}$ & $\mathbf{2 0 0 7}$ & $\mathbf{2 0 0 8}$ & $\mathbf{2 0 0 9}$ & $\mathbf{2 0 1 0}$ & $\mathbf{2 0 1 1}$ & $\mathbf{2 0 1 2}$ & $\mathbf{2 0 1 3}$ \\
\hline 1 & 67.70 & 72.40 & 68.70 & 69.90 & 65.00 & 55.30 & 61.20 & 63.30 & 64.80 & 61.30 \\
\hline 2 & 64.30 & 68.90 & 65.50 & 67.40 & 61.20 & 52.30 & 58.00 & 59.30 & 59.90 & 56.40 \\
\hline 3 & 62.10 & 66.10 & 62.80 & 66.20 & 58.00 & 50.10 & 55.80 & 56.90 & 57.60 & 54.10 \\
\hline 4 & 61.80 & 64.90 & 61.90 & 65.10 & 57.30 & 49.00 & 54.10 & 55.70 & 55.80 & 54.30 \\
\hline 5 & 63.90 & 68.40 & 64.50 & 66.30 & 59.00 & 50.90 & 56.00 & 58.00 & 58.10 & 58.50 \\
\hline 6 & 68.70 & 74.90 & 68.90 & 69.30 & 63.60 & 54.60 & 59.80 & 61.70 & 60.30 & 58.60 \\
\hline 7 & 75.60 & 81.40 & 73.60 & 72.30 & 65.80 & 56.40 & 64.20 & 63.40 & 63.60 & 61.80 \\
\hline 8 & 89.90 & 97.10 & 86.40 & 84.90 & 80.70 & 70.60 & 82.90 & 79.90 & 81.30 & 78.30 \\
\hline 9 & 92.60 & 101.6 & 92.60 & 89.90 & 86.00 & 77.60 & 86.50 & 85.00 & 84.80 & 83.00 \\
\hline 10 & 90.30 & 99.30 & 89.30 & 88.10 & 88.10 & 78.60 & 84.50 & 85.00 & 85.40 & 81.60 \\
\hline 11 & 85.40 & 95.20 & 81.60 & 84.10 & 84.00 & 75.30 & 81.30 & 80.80 & 81.10 & 78.50 \\
\hline 12 & 85.50 & 95.10 & 81.40 & 83.60 & 85.00 & 75.90 & 80.50 & 80.60 & 82.30 & 80.30 \\
\hline 13 & 85.10 & 94.00 & 82.80 & 84.60 & 84.60 & 77.80 & 81.60 & 83.20 & 84.90 & 81.50 \\
\hline 14 & 85.00 & 93.10 & 83.40 & 84.40 & 84.60 & 78.20 & 81.90 & 84.80 & 88.00 & 81.70 \\
\hline 15 & 82.70 & 91.50 & 82.40 & 83.10 & 79.70 & 77.20 & 82.20 & 82.20 & 87.30 & 80.00 \\
\hline 16 & 75.60 & 84.20 & 75.70 & 78.20 & 73.80 & 70.80 & 76.00 & 77.60 & 81.90 & 74.50 \\
\hline 17 & 72.70 & 81.50 & 73.30 & 75.30 & 72.00 & 68.60 & 73.40 & 74.20 & 78.10 & 71.50 \\
\hline 18 & 72.40 & 80.60 & 73.20 & 73.90 & 70.50 & 66.50 & 71.50 & 70.60 & 76.60 & 69.70 \\
\hline 19 & 70.30 & 80.40 & 72.20 & 72.30 & 67.80 & 64.80 & 69.60 & 69.50 & 72.10 & 66.80 \\
\hline 20 & 71.80 & 81.90 & 72.20 & 71.60 & 67.20 & 65.00 & 67.70 & 68.30 & 68.50 & 66.00 \\
\hline 21 & 88.40 & 95.20 & 81.90 & 80.20 & 73.60 & 69.10 & 70.00 & 69.80 & 72.80 & 68.10 \\
\hline 22 & 95.50 & 101.9 & 94.20 & 90.50 & 89.00 & 83.60 & 80.10 & 79.50 & 74.60 & 76.40 \\
\hline 23 & 86.20 & 91.20 & 86.70 & 81.50 & 84.80 & 81.40 & 81.10 & 81.30 & 75.40 & 82.40 \\
\hline 24 & 75.50 & 79.20 & 76.40 & 71.70 & 73.60 & 69.50 & 70.80 & 70.80 & 69.00 & 72.30 \\
\hline
\end{tabular}

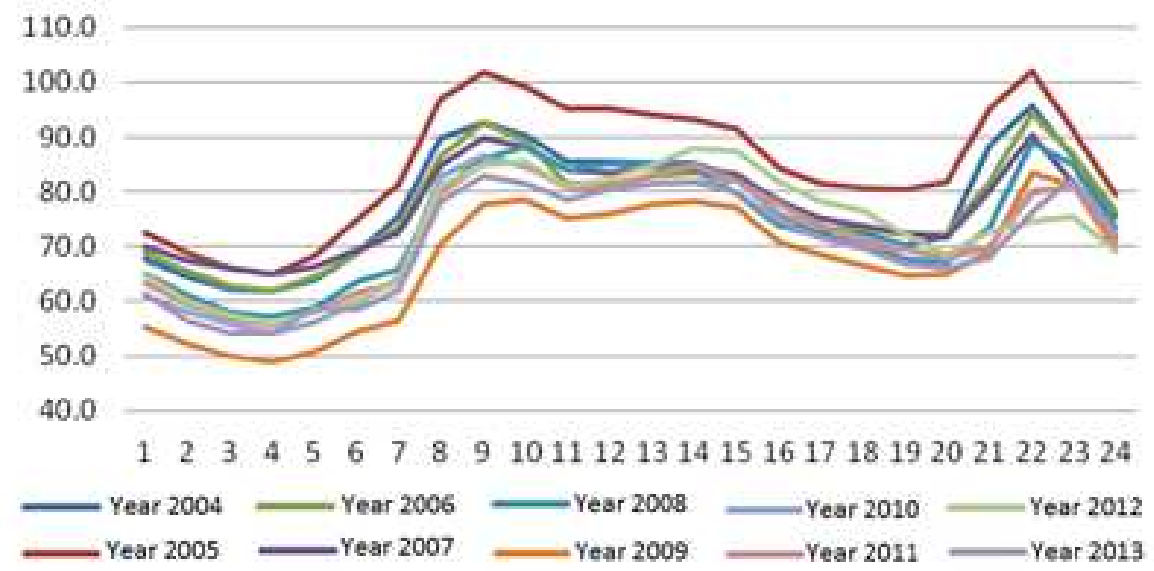

Figure 4: Load curves' variation for the 2004-2013 period [MW]

Table 2 and Figure 5 are presenting the load curves for the 2014-2016 period. They are going to be used in order to validate the forecast based on previously presented load curves (Table 1). 
Table 2: Load curves for 2014-2016 period [MW] - validation period

\begin{tabular}{|c|c|c|c|}
\hline Year/Hour & $\mathbf{2 0 1 4}$ & $\mathbf{2 0 1 5}$ & $\mathbf{2 0 1 6}$ \\
\hline 1 & 62.60 & 65.00 & 71.00 \\
\hline 2 & 57.60 & 59.90 & 65.20 \\
\hline 3 & 56.30 & 58.50 & 63.00 \\
\hline 4 & 55.40 & 57.40 & 61.20 \\
\hline 5 & 55.20 & 58.40 & 62.20 \\
\hline 6 & 58.30 & 58.20 & 64.10 \\
\hline 7 & 60.60 & 60.70 & 67.50 \\
\hline 8 & 78.80 & 78.70 & 82.20 \\
\hline 9 & 84.60 & 83.00 & 87.60 \\
\hline 10 & 85.50 & 84.30 & 87.50 \\
\hline 11 & 81.90 & 80.80 & 85.00 \\
\hline 12 & 83.70 & 80.90 & 86.30 \\
\hline 13 & 84.50 & 82.30 & 87.20 \\
\hline 14 & 86.80 & 83.80 & 87.00 \\
\hline 15 & 84.00 & 84.10 & 86.60 \\
\hline 16 & 78.60 & 79.90 & 80.00 \\
\hline 17 & 75.90 & 79.00 & 79.00 \\
\hline 18 & 75.00 & 74.50 & 77.40 \\
\hline 19 & 71.80 & 71.70 & 76.30 \\
\hline 20 & 71.40 & 71.20 & 74.30 \\
\hline 21 & 74.00 & 72.40 & 74.40 \\
\hline 22 & 87.00 & 88.10 & 89.30 \\
\hline 23 & 81.00 & 83.30 & 84.20 \\
\hline 24 & 69.50 & 75.40 & 76.10 \\
\hline & & & \\
\hline
\end{tabular}

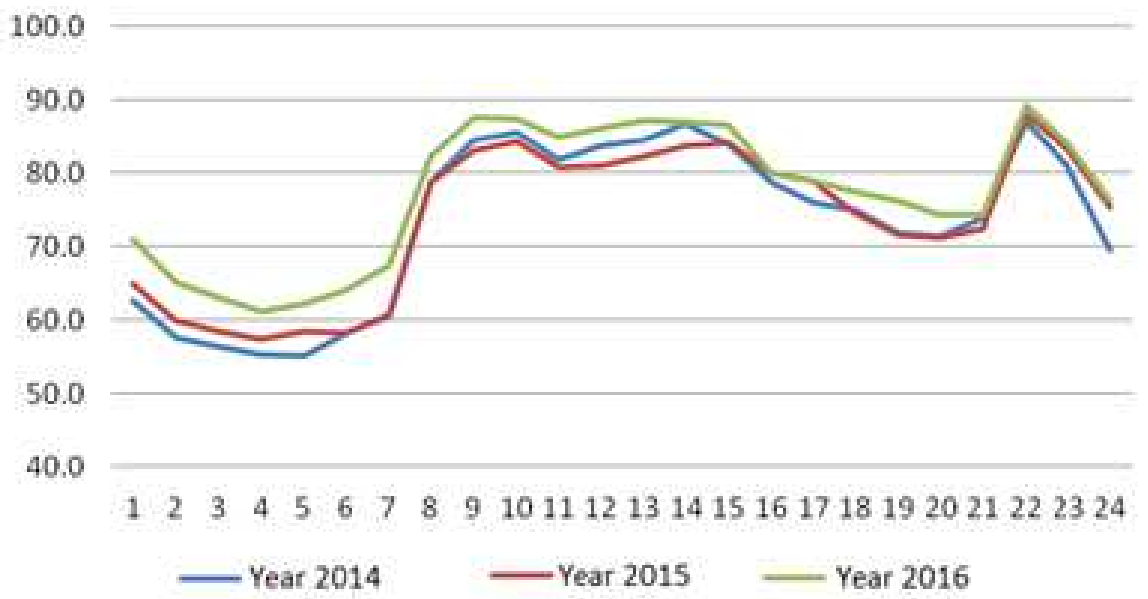

Figure 5: Load curves' variation for the 2014-2016 period [MW]

The following comments are suitable for the case of Tables 1 and 2 (respectively Figure 4 and Figure 5):

- a rigorous consumed power ascending or descending trend is not highlighted, in generally; 
- the load curves are crossing each other (thus, their shape is different), meaning that they are slightly correlated (on horizontally);

- an ascending consumed power trend is highlighted for the 2011-2013 period (with few deviations);

- load curves' correlation degree is reduced (time evolution and daily shape).

The results applying different methods are subjected to:

- forecasted values;

- differences from the known values (relative deviation in \%);

- relative square deviation;

- performance index for each forecasted year (2014-2016). It is computed as the sum of relative square deviations for the 24 hourly values.

These results are presented in Tables 3 - 5 (FV - Forecasted value, RD - Relative deviation, RSD

- Relative square deviation) for the 2014-2016 years and graphically in Figures 6-8.
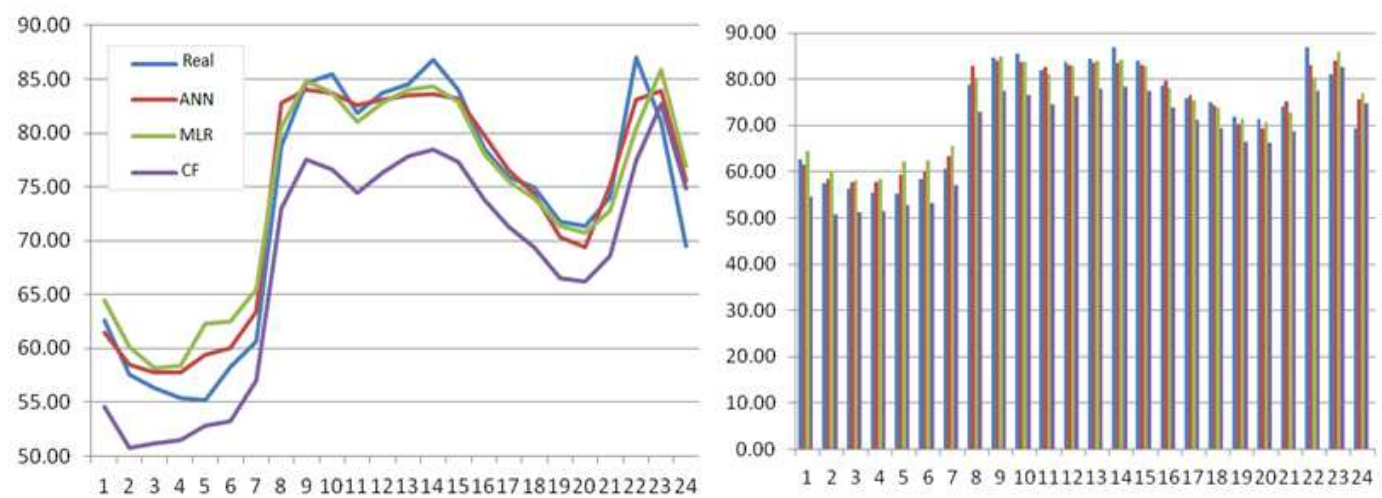

Figure 6: 2014 Forecasted value - comparative analysis [MW]
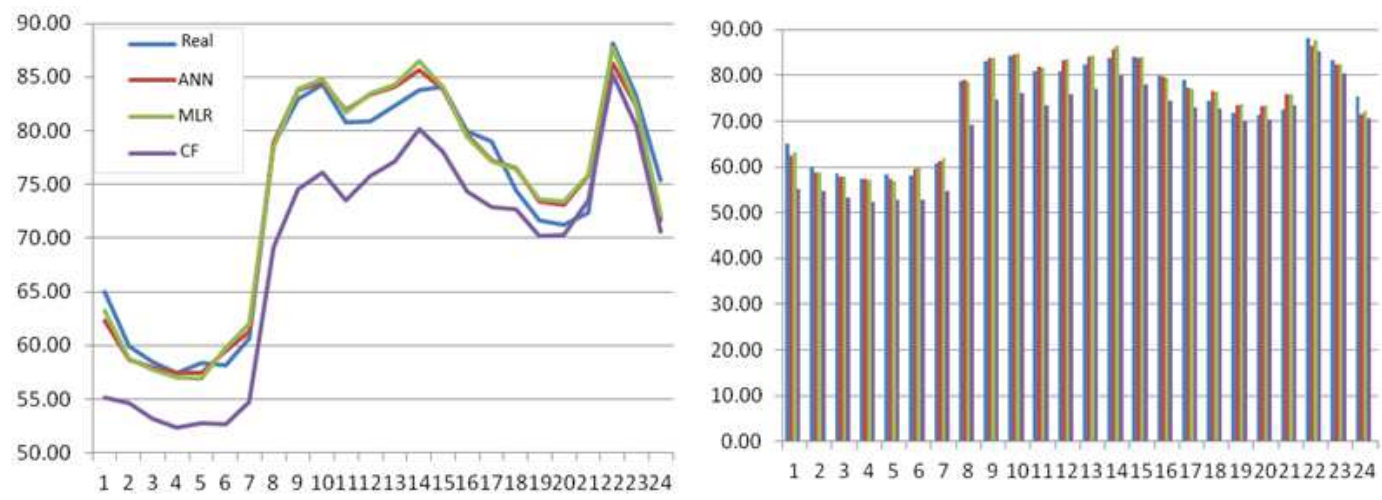

Figure 7: 2015 Forecasted value - comparative analysis [MW]

The performance indices have been gathered in Table 4. The forecast errors are presented in Table 7.

Comparative analysis of the results leads to the following conclusions: 
Table 3: 2014 Year load curves forecasted [MW]

\begin{tabular}{|c|c|c|c|c|c|c|c|c|c|c|}
\hline \multirow{2}{*}{ Hour } & Know & \multicolumn{3}{|c|}{ ANN } & \multicolumn{3}{c|}{ CF } & \multicolumn{3}{c|}{ MLR } \\
\cline { 3 - 11 } & & FV & $\begin{array}{c}\text { RD } \\
{[\%]}\end{array}$ & RSQ & FV & $\begin{array}{c}\text { RD } \\
{[\%]}\end{array}$ & RSQ & FV & $\begin{array}{c}\text { RD } \\
{[\%]}\end{array}$ & RSQ \\
\hline 1 & 62.6 & 61.51 & -1.75 & 3.05 & 54.57 & -12.83 & 164.5 & 64.49 & 3.03 & 9.16 \\
\hline 2 & 57.6 & 58.49 & 1.54 & 2.37 & 50.79 & -11.82 & 139.7 & 60.16 & 4.44 & 19.72 \\
\hline 3 & 56.3 & 57.72 & 2.51 & 6.32 & 51.18 & -9.10 & 82.83 & 58.16 & 3.31 & 10.96 \\
\hline 4 & 55.4 & 57.73 & 4.21 & 17.73 & 51.49 & -7.06 & 49.82 & 58.43 & 5.46 & 29.87 \\
\hline 5 & 55.2 & 59.36 & 7.54 & 56.78 & 52.78 & -4.39 & 19.25 & 62.31 & 12.88 & 165.8 \\
\hline 6 & 58.3 & 59.99 & 2.90 & 8.39 & 53.19 & -8.76 & 76.75 & 62.49 & 7.18 & 51.59 \\
\hline 7 & 60.6 & 63.40 & 4.62 & 21.32 & 57.05 & -5.87 & 34.41 & 65.47 & 8.04 & 64.59 \\
\hline 8 & 78.8 & 82.76 & 5.03 & 25.28 & 73.04 & -7.31 & 53.46 & 80.48 & 2.14 & 4.56 \\
\hline 9 & 84.6 & 84.05 & -0.66 & 0.43 & 77.52 & -8.37 & 70.09 & 84.85 & 0.30 & 0.09 \\
\hline 10 & 85.5 & 83.76 & -2.03 & 4.13 & 76.62 & -10.38 & 107.8 & 83.71 & -2.09 & 4.37 \\
\hline 11 & 81.9 & 82.53 & 0.77 & 0.60 & 74.48 & -9.06 & 82.07 & 81.03 & -1.06 & 1.12 \\
\hline 12 & 83.7 & 83.13 & -0.68 & 0.46 & 76.32 & -8.82 & 77.78 & 82.78 & -1.10 & 1.20 \\
\hline 13 & 84.5 & 83.52 & -1.16 & 1.36 & 77.82 & -7.91 & 62.57 & 83.99 & -0.60 & 0.37 \\
\hline 14 & 86.8 & 83.60 & -3.69 & 13.59 & 78.44 & -9.64 & 92.83 & 84.29 & -2.89 & 8.33 \\
\hline 15 & 84.0 & 83.18 & -0.98 & 0.96 & 77.37 & -7.90 & 62.35 & 82.88 & -1.33 & 1.77 \\
\hline 16 & 78.6 & 79.76 & 1.48 & 2.19 & 73.81 & -6.09 & 37.1 & 78.03 & -0.72 & 0.52 \\
\hline 17 & 75.9 & 76.47 & 0.75 & 0.56 & 71.22 & -6.17 & 38.03 & 75.43 & -0.62 & 0.38 \\
\hline 18 & 75.0 & 74.39 & -0.81 & 0.66 & 69.41 & -7.45 & 55.54 & 73.91 & -1.45 & 2.10 \\
\hline 19 & 71.8 & 70.36 & -2.01 & 4.03 & 66.54 & -7.32 & 53.61 & 71.4 & -0.56 & 0.31 \\
\hline 20 & 71.4 & 69.39 & -2.81 & 7.92 & 66.17 & -7.32 & 53.64 & 70.78 & -0.87 & 0.76 \\
\hline 21 & 74.0 & 75.17 & 1.59 & 2.52 & 68.62 & -7.27 & 52.82 & 72.78 & -1.65 & 2.73 \\
\hline 22 & 87.0 & 83.09 & -4.50 & 20.21 & 77.46 & -10.96 & 120.2 & 80.38 & -7.60 & 57.82 \\
\hline 23 & 81.0 & 83.95 & 3.65 & 13.29 & 82.64 & 2.03 & 4.12 & 85.93 & 6.08 & 36.99 \\
\hline 24 & 69.5 & 75.60 & 8.78 & 77.11 & 74.84 & 7.69 & 59.13 & 76.92 & 10.68 & 113.9 \\
\hline & & $P I_{2014}$ & & 291.3 & $P I_{2014}$ & & 1650 & $P I_{2014}$ & 589.1 \\
\hline
\end{tabular}
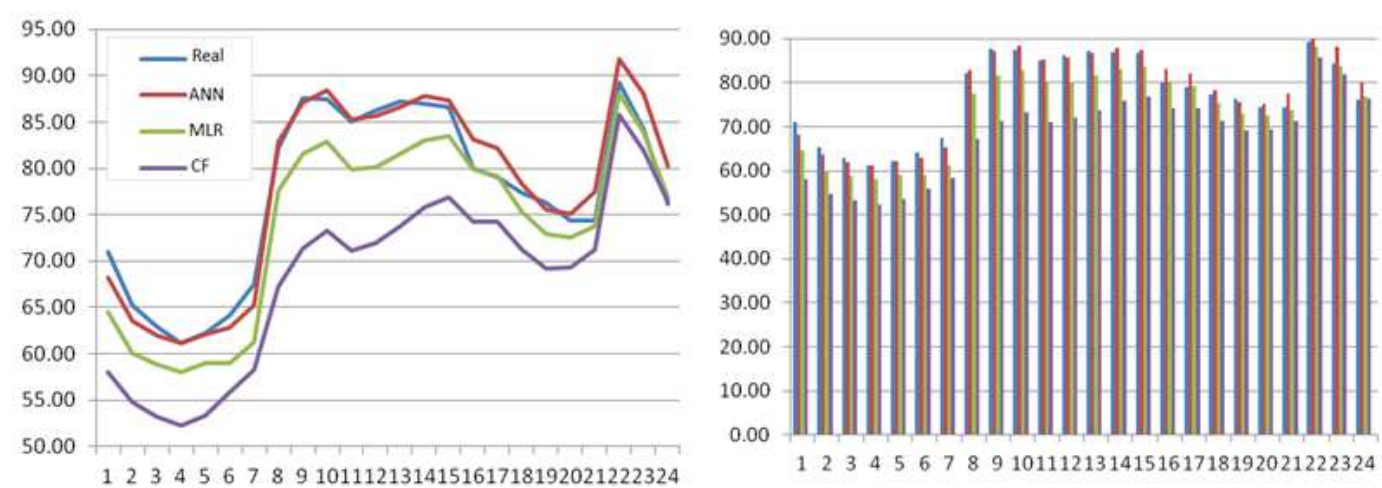

Figure 8: 2016 Forecasted value - comparative analysis [MW]

- classical forecasting methods (CF, MLR) are inadequate. The global performance index (Table 6) sustains this fact;

- ANN method: the best case is recorded for the 2015 year (Table 6: performance index 
Table 4: 2015 Year load curves forecasted [MW]

\begin{tabular}{|c|c|c|c|c|c|c|c|c|c|c|}
\hline \multirow{2}{*}{ Hour } & Know & \multicolumn{3}{|c|}{ ANN } & \multicolumn{3}{c|}{ CF } & \multicolumn{3}{c|}{ MLR } \\
\cline { 3 - 11 } & & FV & $\begin{array}{c}\text { RD } \\
\text { [\%] }\end{array}$ & RSQ & FV & $\begin{array}{c}\text { RD } \\
{[\%]}\end{array}$ & RSQ & FV & $\begin{array}{c}\text { RD } \\
{[\%]}\end{array}$ & RSQ \\
\hline 1 & 65.0 & 62.36 & -4.05 & 16.44 & 55.14 & -15.17 & 230.1 & 63.23 & -2.72 & 7.42 \\
\hline 2 & 59.9 & 58.70 & -2.01 & 4.03 & 54.67 & -8.73 & 76.23 & 58.83 & -1.79 & 3.20 \\
\hline 3 & 58.5 & 57.94 & -0.95 & 0.90 & 53.22 & -9.03 & 81.46 & 57.75 & -1.28 & 1.63 \\
\hline 4 & 57.4 & 57.47 & 0.13 & 0.02 & 52.40 & -8.71 & 75.88 & 57.03 & -0.64 & 0.40 \\
\hline 5 & 58.4 & 57.42 & -1.67 & 2.79 & 52.80 & -9.59 & 91.95 & 56.95 & -2.49 & 6.20 \\
\hline 6 & 58.2 & 59.50 & 2.23 & 4.97 & 52.70 & -9.45 & 89.31 & 59.83 & 2.80 & 7.84 \\
\hline 7 & 60.7 & 61.31 & 1.00 & 1.00 & 54.70 & -9.88 & 97.71 & 62.00 & 2.14 & 4.57 \\
\hline 8 & 78.7 & 78.94 & 0.30 & 0.09 & 69.18 & -12.10 & 146.5 & 78.49 & -0.26 & 0.07 \\
\hline 9 & 83.0 & 83.78 & 0.94 & 0.88 & 74.59 & -10.13 & 102.6 & 83.85 & 1.02 & 1.05 \\
\hline 10 & 84.3 & 84.49 & 0.23 & 0.05 & 76.14 & -9.68 & 93.74 & 84.80 & 0.59 & 0.35 \\
\hline 11 & 80.8 & 81.92 & 1.39 & 1.93 & 73.56 & -8.96 & 80.22 & 81.70 & 1.11 & 1.23 \\
\hline 12 & 80.9 & 83.40 & 3.09 & 9.56 & 75.76 & -6.36 & 40.40 & 83.45 & 3.16 & 9.97 \\
\hline 13 & 82.3 & 84.06 & 2.14 & 4.57 & 77.16 & -6.25 & 39.02 & 84.31 & 2.45 & 5.98 \\
\hline 14 & 83.8 & 85.64 & 2.20 & 4.84 & 80.13 & -4.38 & 19.19 & 86.52 & 3.25 & 10.57 \\
\hline 15 & 84.1 & 83.88 & -0.26 & 0.07 & 78.05 & -7.19 & 51.75 & 84.15 & 0.05 & 0.00 \\
\hline 16 & 79.9 & 79.64 & -0.33 & 0.11 & 74.34 & -6.96 & 48.51 & 79.42 & -0.60 & 0.36 \\
\hline 17 & 79.0 & 77.24 & -2.22 & 4.94 & 72.87 & -7.76 & 60.21 & 77.12 & -2.38 & 5.68 \\
\hline 18 & 74.5 & 76.49 & 2.67 & 7.13 & 72.72 & -2.40 & 5.74 & 76.43 & 2.59 & 6.72 \\
\hline 19 & 71.7 & 73.39 & 2.36 & 5.58 & 70.15 & -2.16 & 4.65 & 73.67 & 2.75 & 7.56 \\
\hline 20 & 71.2 & 73.11 & 2.68 & 7.18 & 70.29 & -1.28 & 1.63 & 73.43 & 3.13 & 9.81 \\
\hline 21 & 72.4 & 75.83 & 4.74 & 22.5 & 73.56 & 1.60 & 2.56 & 75.89 & 4.82 & 23.24 \\
\hline 22 & 88.1 & 86.31 & -2.03 & 4.12 & 85.27 & -3.21 & 10.32 & 87.72 & -0.43 & 0.18 \\
\hline 23 & 83.3 & 82.35 & -1.14 & 1.30 & 80.44 & -3.43 & 11.77 & 82.46 & -1.01 & 1.01 \\
\hline 24 & 75.4 & 71.64 & -4.98 & 24.83 & 70.66 & -6.28 & 39.46 & 72.24 & -4.20 & 17.61 \\
\hline & & $P I_{2015}$ & & 129.8 & $P I_{2015}$ & & 1500 & $P I_{2015}$ & & 132.7 \\
\hline
\end{tabular}

129.8); the worst case is recorded for the 2014 year (Table 6: performance index 291.3);

- the forecast errors are ranging between $4.7 \%$ and $8.8 \%$ in case of ANN based method. Values of $18 \%$, respectively $13 \%$ have been obtained in case of CF, respectively MLR.

The load curves for a period of 10 years (2004-2013) are presented in Table 8, for the most significant summer day. These ones are corresponding to the entire network assembly of the considered distribution network operator. These data are going to be used in order to perform the forecast for 2014-2016 period.

The same information is graphically presented in Figure 9.

The load curves for 3 years period are presented in Table 9 and Figure 10. These ones are used in order to validate the performed forecast.

The trend is unclear for the 2004-2013 period. There are several increasing periods, alternating with decreasing ones, during a relatively short set of values. The load curves are not crossing each other. This means that they are characterized by a high correlation degree.

The 2014-2016 period is also characterized by an unclear evolution. The load curves correlation degree during a day is relatively good, taking into consideration their shape. Thus, it is envisaged that the ANN based forecasting methods could lead to better results. 
Table 5: 2016 Year load curves forecasted [MW]

\begin{tabular}{|c|c|c|c|c|c|c|c|c|c|c|}
\hline \multirow{2}{*}{ Hour } & \multirow{2}{*}{$\begin{array}{c}\text { Know } \\
\text { value }\end{array}$} & \multicolumn{3}{|c|}{ ANN } & \multicolumn{3}{c|}{ CF } & \multicolumn{3}{c|}{ MLR } \\
\cline { 3 - 11 } & & FV & $\begin{array}{c}\text { RD } \\
{[\%]}\end{array}$ & RSQ & FV & $\begin{array}{c}\text { RD } \\
{[\%]}\end{array}$ & RSQ & FV & $\begin{array}{c}\text { RD } \\
{[\%]}\end{array}$ & RSQ \\
\hline 1 & 71.0 & 68.29 & -3.82 & 14.57 & 58.06 & -18.23 & 332.2 & 64.50 & -9.15 & 83.75 \\
\hline 2 & 65.2 & 63.51 & -2.60 & 6.74 & 54.80 & -15.95 & 254.4 & 60.05 & -7.91 & 62.49 \\
\hline 3 & 63.0 & 62.00 & -1.58 & 2.51 & 53.22 & -15.52 & 240.9 & 58.90 & -6.51 & 42.37 \\
\hline 4 & 61.2 & 61.12 & -0.12 & 0.02 & 52.20 & -14.71 & 216.3 & 58.02 & -5.20 & 27.01 \\
\hline 5 & 62.2 & 62.17 & -0.05 & 0.00 & 53.40 & -14.15 & 200.2 & 59.02 & -5.11 & 26.11 \\
\hline 6 & 64.1 & 62.85 & -1.95 & 3.79 & 55.82 & -12.92 & 166.9 & 58.95 & -8.04 & 64.58 \\
\hline 7 & 67.5 & 65.21 & -3.39 & 11.52 & 58.25 & -13.70 & 187.8 & 61.30 & -9.19 & 84.40 \\
\hline 8 & 82.2 & 82.93 & 0.88 & 0.78 & 67.25 & -18.19 & 330.9 & 77.56 & -5.64 & 31.86 \\
\hline 9 & 87.6 & 87.15 & -0.52 & 0.27 & 71.34 & -18.56 & 344.4 & 81.56 & -6.89 & 47.51 \\
\hline 10 & 87.5 & 88.45 & 1.08 & 1.18 & 73.25 & -16.29 & 265.3 & 82.88 & -5.28 & 27.87 \\
\hline 11 & 85.0 & 85.3 & 0.36 & 0.13 & 71.12 & -16.33 & 266.8 & 79.89 & -6.01 & 36.08 \\
\hline 12 & 86.3 & 85.62 & -0.79 & 0.63 & 71.94 & -16.64 & 276.9 & 80.13 & -7.15 & 51.08 \\
\hline 13 & 87.2 & 86.65 & -0.63 & 0.40 & 73.79 & -15.38 & 236.5 & 81.54 & -6.50 & 42.19 \\
\hline 14 & 87.0 & 87.79 & 0.90 & 0.81 & 75.82 & -12.86 & 165.3 & 83.03 & -4.56 & 20.79 \\
\hline 15 & 86.6 & 87.29 & 0.80 & 0.64 & 76.85 & -11.25 & 126.7 & 83.46 & -3.63 & 13.19 \\
\hline 16 & 80.0 & 83.18 & 3.98 & 15.80 & 74.21 & -7.24 & 52.36 & 79.84 & -0.20 & 0.04 \\
\hline 17 & 79.0 & 82.21 & 4.06 & 16.52 & 74.26 & -6.00 & 36.01 & 79.18 & 0.23 & 0.05 \\
\hline 18 & 77.4 & 78.34 & 1.22 & 1.48 & 71.28 & -7.90 & 62.45 & 75.29 & -2.73 & 7.45 \\
\hline 19 & 76.3 & 75.57 & -0.96 & 0.91 & 69.18 & -9.33 & 87.04 & 72.91 & -4.44 & 19.73 \\
\hline 20 & 74.3 & 75.12 & 1.11 & 1.22 & 69.31 & -6.71 & 45.06 & 72.59 & -2.30 & 5.28 \\
\hline 21 & 74.4 & 77.49 & 4.16 & 17.30 & 71.25 & -4.24 & 17.97 & 73.80 & -0.81 & 0.65 \\
\hline 22 & 89.3 & 91.78 & 2.78 & 7.71 & 85.83 & -3.89 & 15.13 & 88.02 & -1.43 & 2.06 \\
\hline 23 & 84.2 & 88.10 & 4.64 & 21.5 & 81.88 & -2.75 & 7.57 & 83.87 & -0.39 & 0.15 \\
\hline 24 & 76.1 & 80.20 & 5.39 & 29.02 & 76.35 & 0.33 & 0.11 & 76.94 & 1.10 & 1.21 \\
\hline & & $P I_{2016}$ & & 155.5 & $P I_{2016}$ & & 3935 & $P I_{2016}$ & 697.9 \\
\hline
\end{tabular}

Table 6: Performance indices - comparative analysis

\begin{tabular}{|c|c|c|c|}
\hline & ANN & CF & MLR \\
\hline$P I_{2014}$ & 291.3 & 1650 & 589.1 \\
\hline$P I_{2015}$ & 129.8 & 1500 & 132.7 \\
\hline$P I_{2016}$ & 155.5 & 3935 & 697.9 \\
\hline$P I_{\text {total }}$ & 576.6 & 7085 & 1420 \\
\hline
\end{tabular}

Table 7: Maximum forecast errors

\begin{tabular}{|c|c|c|c|}
\hline & ANN & CF & MLR \\
\hline 2014 & 8.78 & 12.83 & 12.88 \\
\hline 2015 & 4.74 & 15.17 & 4.82 \\
\hline 2016 & 5.39 & 18.23 & 8.19 \\
\hline Maximum & 8.78 & 18.23 & 12.88 \\
\hline
\end{tabular}

The same forecasting methods have been applied (as the ones presented in Tables 2-5). The 
Table 8: Load curves for 2004-2013 period [MW]

\begin{tabular}{|c|c|c|c|c|c|c|c|c|c|c|}
\hline Year/Hour & $\mathbf{2 0 0 4}$ & $\mathbf{2 0 0 5}$ & $\mathbf{2 0 0 6}$ & $\mathbf{2 0 0 7}$ & $\mathbf{2 0 0 8}$ & $\mathbf{2 0 0 9}$ & $\mathbf{2 0 1 0}$ & $\mathbf{2 0 1 1}$ & $\mathbf{2 0 1 2}$ & $\mathbf{2 0 1 3}$ \\
\hline 1 & 455.5 & 460.2 & 472.1 & 436.3 & 418.7 & 398.4 & 411.6 & 401.7 & 401.7 & 388.6 \\
\hline 2 & 427.8 & 452.8 & 457.7 & 427.3 & 415.0 & 382.6 & 390.8 & 381.4 & 380.6 & 372.2 \\
\hline 3 & 417.9 & 441.0 & 436.1 & 421.0 & 410.6 & 378.5 & 394.7 & 386.6 & 389.0 & 387.8 \\
\hline 4 & 413.9 & 454.8 & 447.6 & 418.4 & 393.8 & 365.7 & 382.7 & 374.7 & 385.8 & 384.1 \\
\hline 5 & 434.4 & 456.8 & 459.3 & 424.5 & 404.1 & 380.7 & 403.8 & 391.4 & 389.8 & 376.5 \\
\hline 6 & 459.4 & 465.2 & 469.0 & 441.7 & 411.6 & 388.1 & 403.5 & 404.8 & 382.1 & 358.6 \\
\hline 7 & 477.9 & 503.0 & 489.1 & 455.6 & 422.1 & 396.2 & 395.5 & 404.5 & 393.6 & 377.7 \\
\hline 8 & 534.9 & 570.2 & 517.9 & 525.6 & 473.9 & 449.2 & 464.4 & 446.3 & 437.0 & 432.3 \\
\hline 9 & 554.1 & 607.2 & 529.7 & 574.0 & 525.4 & 479.5 & 481.7 & 489.0 & 483.4 & 486.0 \\
\hline 10 & 536.9 & 604.8 & 530.5 & 564.6 & 530.8 & 492.4 & 490.5 & 506.5 & 494.3 & 479.4 \\
\hline 11 & 519.1 & 583.5 & 499.0 & 549.3 & 529.6 & 483.5 & 472.7 & 492.0 & 490.5 & 487.8 \\
\hline 12 & 512.3 & 557.3 & 527.7 & 531.5 & 514.1 & 477.6 & 465.7 & 469.2 & 472.6 & 474.8 \\
\hline 13 & 496.2 & 551.3 & 539.4 & 525.2 & 500.5 & 468.6 & 445.1 & 467.3 & 464.9 & 460.9 \\
\hline 14 & 502.0 & 567.3 & 547.0 & 518.9 & 495.3 & 473.4 & 452.3 & 489.4 & 472.0 & 456.7 \\
\hline 15 & 491.1 & 546.9 & 529.1 & 518.6 & 488.2 & 462.2 & 438.2 & 463.9 & 467.6 & 458.5 \\
\hline 16 & 452.2 & 505.2 & 485.6 & 487.7 & 451.3 & 450.6 & 447.0 & 451.2 & 451.7 & 428.9 \\
\hline 17 & 462.9 & 490.6 & 492.0 & 479.3 & 439.1 & 442.8 & 440.0 & 449.3 & 443.6 & 426.8 \\
\hline 18 & 448.5 & 502.7 & 490.6 & 476.5 & 424.6 & 443.0 & 445.4 & 445.5 & 440.9 & 414.9 \\
\hline 19 & 440.4 & 495.6 & 488.9 & 464.3 & 405.2 & 432.4 & 419.4 & 453.3 & 443.5 & 413.5 \\
\hline 20 & 436.8 & 476.2 & 445.0 & 442.2 & 393.6 & 418.9 & 392.2 & 418.8 & 427.2 & 409.2 \\
\hline 21 & 510.1 & 534.7 & 496.2 & 479.5 & 426.3 & 421.5 & 386.5 & 405.2 & 432.0 & 424.6 \\
\hline 22 & 545.3 & 561.3 & 534.7 & 528.4 & 494.5 & 478.9 & 432.7 & 429.4 & 445.7 & 453.3 \\
\hline 23 & 562.8 & 580.5 & 573.5 & 540.3 & 524.9 & 518.9 & 498.9 & 475.3 & 463.7 & 480.3 \\
\hline 24 & 536.7 & 538.0 & 524.1 & 501.8 & 488.1 & 482.1 & 443.3 & 444.7 & 437.5 & 438.7 \\
\hline
\end{tabular}

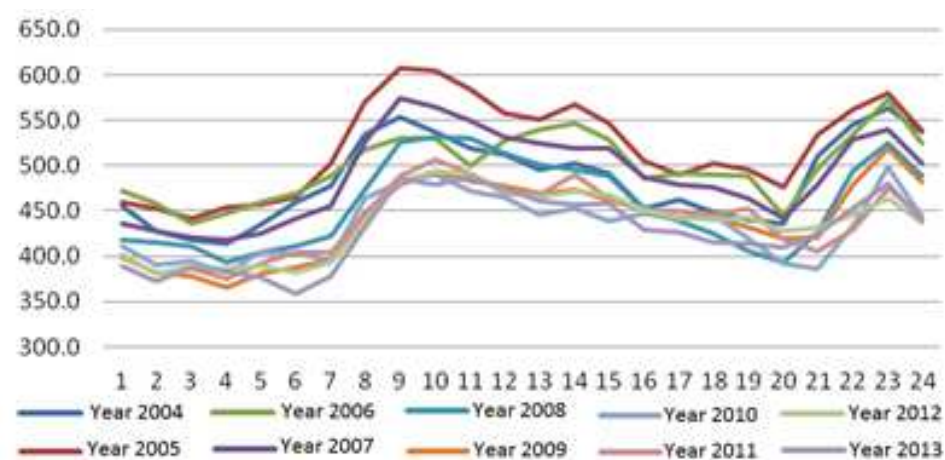

Figure 9: Known load curves for 2004-2013 period

extended results are not presented anymore. Only a synthesis of the forecasted values is presented in Table 10. The same results are graphically presented in Figures 11-13. The performance indices are synthesized in Table 11.

The conventional forecasting methods (CF and MLR) are totally inadequate (Table 11, global performance indices values). Among them, the MLR provides more accurate results.

Comparing the yearly performance indices for the ANN based results it is highlighting that the 2016 year the best situation is recorded (around 59). The worst one is recorded for the 2014 
Table 9: Load curves for validation period 2014-2016 [MW]

\begin{tabular}{|c|c|c|c|}
\hline Year/Hour & $\mathbf{2 0 1 4}$ & $\mathbf{2 0 1 5}$ & $\mathbf{2 0 1 6}$ \\
\hline 1 & 389.4 & 384.8 & 384.2 \\
\hline 2 & 374.7 & 370.3 & 364.6 \\
\hline 3 & 377.4 & 380.9 & 373.9 \\
\hline 4 & 370.8 & 364.9 & 363.3 \\
\hline 5 & 370.6 & 367.4 & 372.5 \\
\hline 6 & 368.2 & 358.1 & 364.3 \\
\hline 7 & 382.0 & 381.3 & 380.1 \\
\hline 8 & 430.4 & 421.4 & 422.7 \\
\hline 9 & 464.1 & 450.9 & 449.9 \\
\hline 10 & 482.8 & 472.9 & 471.2 \\
\hline 11 & 490.3 & 486.2 & 481.9 \\
\hline 12 & 460.6 & 453.9 & 452.7 \\
\hline 13 & 449.0 & 442.3 & 450.7 \\
\hline 14 & 459.8 & 454.9 & 450.5 \\
\hline 15 & 453.0 & 450.5 & 441.4 \\
\hline 16 & 433.3 & 431.1 & 430.0 \\
\hline 17 & 437.5 & 440.2 & 433.8 \\
\hline 18 & 427.2 & 418.0 & 423.0 \\
\hline 19 & 419.9 & 409.6 & 415.9 \\
\hline 20 & 408.3 & 402.2 & 405.6 \\
\hline 21 & 410.9 & 411.6 & 406.0 \\
\hline 22 & 450.0 & 439.8 & 436.4 \\
\hline 23 & 472.6 & 474.6 & 467.7 \\
\hline 24 & 438.6 & 439.4 & 434.6 \\
\hline & & & \\
\hline
\end{tabular}

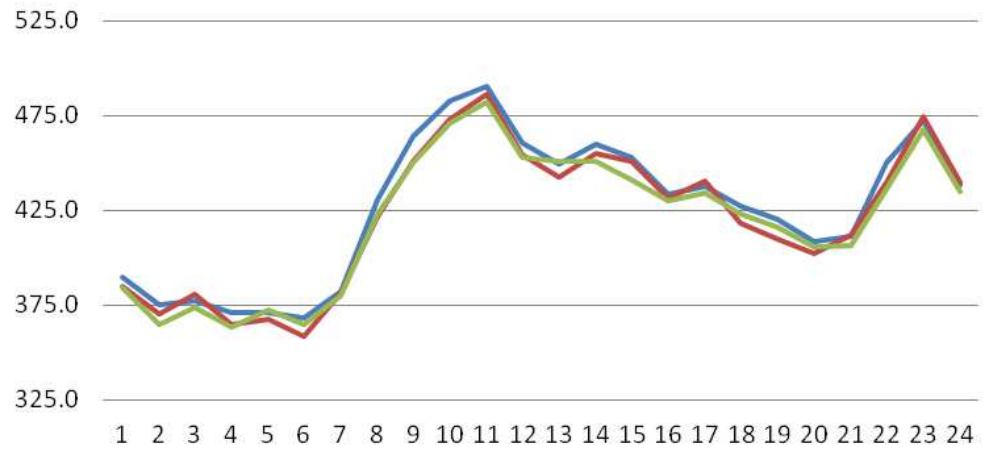

Figure 10: Load curves corresponding to the forecasted period

year (around 152).

The maximum forecast errors are presented in Table 12 .

Based on the results presented in Table 12, acceptable values have been obtained for ANN based forecasting method (bellow $5 \%$ errors). In case of CF and MLR forecasting based methods, they are ranging towards $17 \%$ respectively $8 \%$.

The considered distribution system operator is divided into a number of 4 distribution branches. A comparison between the obtained values is performed in the following. Only the results corresponding to the ANN and MLR based forecasting methods have been considered. 
Table 10: Performance indices - comparative analysis

\begin{tabular}{|l|l|l|l|l|l|l|l|l|}
\hline \multicolumn{3}{|c|}{2014} & \multicolumn{3}{c|}{2015} & \multicolumn{3}{|c|}{2016} \\
\hline ANN & CF & MLR & ANN & CF & MLR & ANN & CF & MLR \\
\hline 387.7 & 358.8 & 365.2 & 365.8 & 349.6 & 357.7 & 379.9 & 343.5 & 353.8 \\
\hline 382.5 & 355.6 & 353.1 & 361.0 & 342.8 & 347.2 & 366.1 & 341.0 & 343.3 \\
\hline 386.1 & 354.2 & 368.3 & 367.3 & 343.7 & 351.4 & 377.0 & 343.2 & 354.1 \\
\hline 385.7 & 353.5 & 367.1 & 367.5 & 342.2 & 347.7 & 362.0 & 339.7 & 342.2 \\
\hline 384.1 & 351.7 & 362.5 & 369.8 & 344.2 & 349.4 & 364.7 & 341.2 & 346.1 \\
\hline 380.6 & 348.4 & 349.1 & 368.5 & 341.2 & 349.2 & 356.2 & 338.6 & 339.9 \\
\hline 386.2 & 348.7 & 367.1 & 378.1 & 343.2 & 362.8 & 379.0 & 339.8 & 361.5 \\
\hline 421.7 & 375.9 & 415.6 & 411.1 & 353.6 & 405.9 & 418.0 & 349.6 & 397.6 \\
\hline 477.3 & 438.8 & 463.5 & 446.8 & 390.8 & 436.6 & 442.5 & 383.3 & 424.9 \\
\hline 472.0 & 437.3 & 460.3 & 474.7 & 423.5 & 454.9 & 457.4 & 399.4 & 445.8 \\
\hline 476.4 & 441.2 & 469.8 & 486.6 & 426.5 & 463.7 & 464.9 & 418.6 & 459.5 \\
\hline 468.5 & 438.5 & 461.2 & 457.1 & 398.5 & 441.0 & 445.8 & 398.4 & 434.2 \\
\hline 456.9 & 437.4 & 451.7 & 446.4 & 394.8 & 433.5 & 437.5 & 392.4 & 426.4 \\
\hline 454.4 & 432.2 & 450.4 & 458.5 & 407.2 & 444.9 & 447.3 & 407.3 & 439.3 \\
\hline 454.4 & 413.3 & 454.2 & 451.7 & 407.2 & 441.5 & 444.6 & 408.5 & 437.7 \\
\hline 419.7 & 390.9 & 431.2 & 429.8 & 393.8 & 427.1 & 430.0 & 392.5 & 423.3 \\
\hline 419.2 & 393.0 & 431.5 & 431.8 & 402.3 & 432.9 & 437.9 & 406.5 & 433.1 \\
\hline 409.9 & 384.0 & 423.5 & 422.0 & 396.1 & 426.3 & 419.6 & 388.3 & 416.2 \\
\hline 409.2 & 386.9 & 424.3 & 415.3 & 392.5 & 422.3 & 412.4 & 384.8 & 411.0 \\
\hline 402.5 & 387.6 & 422.7 & 406.8 & 386.1 & 414.6 & 405.8 & 381.6 & 406.7 \\
\hline 420.3 & 407.8 & 437.9 & 410.0 & 392.3 & 418.9 & 414.8 & 394.2 & 416.7 \\
\hline 454.9 & 440.6 & 464.5 & 443.8 & 435.0 & 454.1 & 438.6 & 425.4 & 442.7 \\
\hline 485.9 & 461.3 & 489.7 & 464.1 & 452.8 & 475.6 & 461.6 & 453.6 & 474.6 \\
\hline 439.7 & 436.9 & 456.6 & 432.2 & 433.9 & 449.1 & 439.1 & 433.5 & 446.8 \\
\hline
\end{tabular}

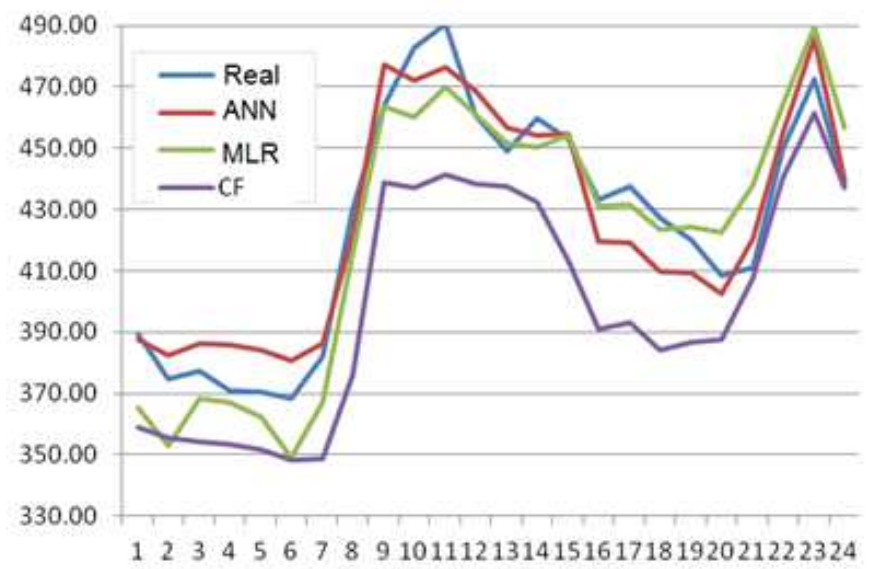

Figure 11: 2014 year forecasted load curve

The specific performance indices have been synthesized in Table 13. These indices have been obtained by dividing the performance index with 24 ( 3 years $\mathrm{x} 24$ hours $=72$ ).

The distribution system operator entire network assembly is ranking on the $1^{\text {st }}$ place. It is a logic result: the errors are attenuating through summation, due to the opposite signs. It closely 


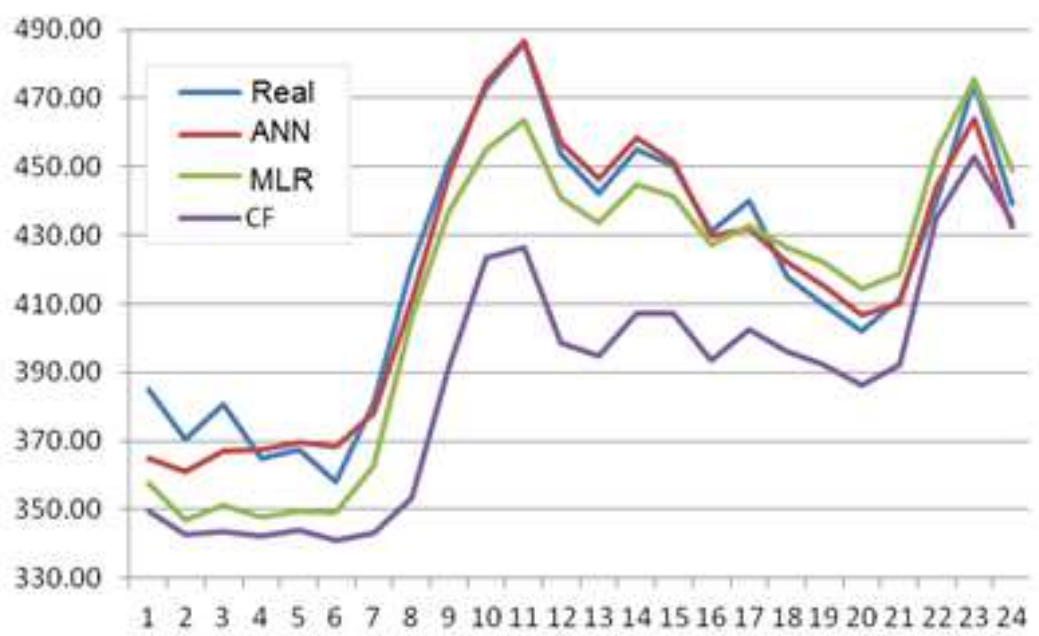

Figure 12: 2015 year forecasted load curve

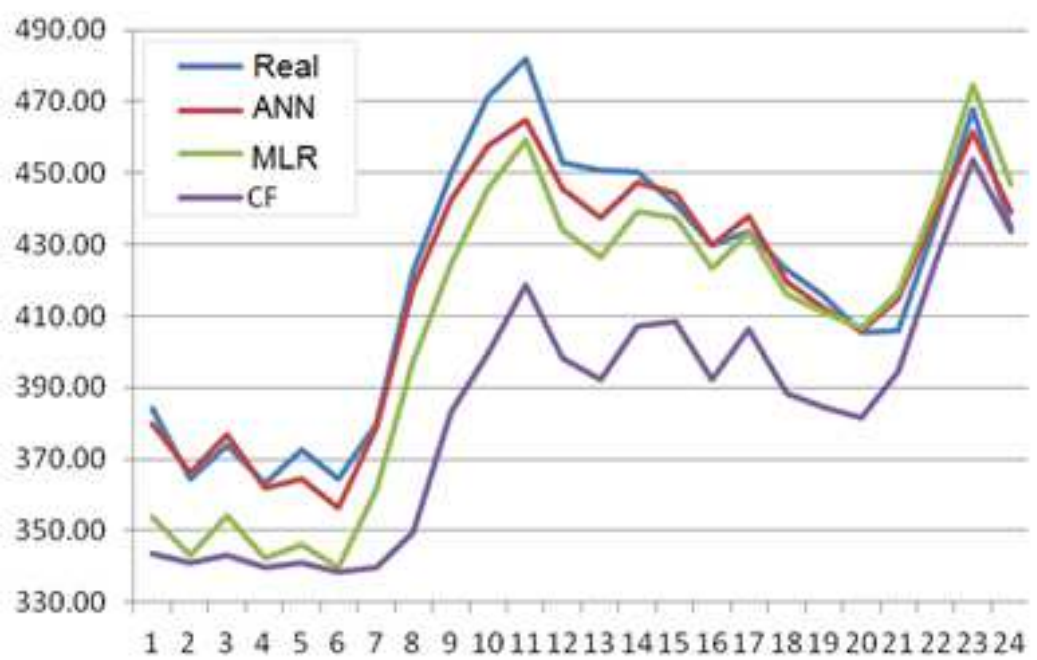

Figure 13: 2016 year forecasted load curve

Table 11: Performance indices - comparative analysis

\begin{tabular}{|c|c|c|c|}
\hline & ANN & CF & MLR \\
\hline$P I_{2014}$ & 151.5 & 1200 & 281.0 \\
\hline$P I_{2015}$ & 78.89 & 1855 & 350.9 \\
\hline$P I_{2016}$ & 58.72 & 2176 & 473.1 \\
\hline$P I_{\text {total }}$ & 289.1 & 5231 & 1105 \\
\hline
\end{tabular}

followed up by Distribution Branch TM and, at a considered distance, by Distribution Branch AR and Distribution Branch HD. Distribution Branch CS is ranking on the last place.

The Distribution Branch TM is ranking on the $1^{\text {st }}$ place, if the MLR based results are discussed. The entire network assembly is following up. The Distribution Branches AR and HD are the next ones. The Distribution Network CS is also ranking on the last place. The $1^{\text {st }}$ two places have been changed comparing with the ANN based hierarchy. The ANN specific indices 
Table 12: Maximum forecast errors

\begin{tabular}{|c|c|c|c|}
\hline & ANN & CF & MLR \\
\hline 2014 & 4.17 & 12.67 & 6.56 \\
\hline 2015 & 4.94 & 16.09 & 7.75 \\
\hline 2016 & 3.52 & 17.30 & 7.90 \\
\hline Maximum & 4.94 & 17.30 & 7.90 \\
\hline
\end{tabular}

Table 13: Specific performance indices

\begin{tabular}{|c|l|c|c|c|c|c|}
\hline \multirow{2}{*}{ No } & \multirow{2}{*}{ Distribution branch } & \multicolumn{2}{|c|}{ ANN } & \multicolumn{2}{c|}{ MLR } & \multirow{2}{*}{ Specific indices ratio } \\
\cline { 3 - 6 } 1 & $\begin{array}{l}\text { Entire } \\
\text { network assembly }\end{array}$ & 298.1 & 4.02 & 1105 & 15.35 & 3.82 \\
\hline 2 & $\begin{array}{l}\text { Distribution } \\
\text { branch TM }\end{array}$ & 354.1 & 4.92 & 958.5 & 13.31 & 2.71 \\
\hline 3 & $\begin{array}{l}\text { Distribution } \\
\text { branch AR }\end{array}$ & 576.6 & 8.01 & 1420 & 19.72 & 2.46 \\
\hline 4 & $\begin{array}{l}\text { Distribution } \\
\text { branch HD }\end{array}$ & 821.5 & 11.41 & 2690 & 37.36 & 3.27 \\
\hline 5 & $\begin{array}{l}\text { Distribution } \\
\text { branch CS }\end{array}$ & 2278 & 31.64 & 10530 & 146.2 & 4.63 \\
\hline
\end{tabular}

are covering a large domain, from 4.02 (entire network assembly), to 31.64 (Distribution Branch CS). Their values are influenced by the input data (2004-2013), the load curves' correlation degree and the real consumption evolution (2014-2016).

High values for the specific performance index highlight the presence of possible wrong load consumption data (discrepancy is recorded). An acceptable situation could be obtained if they are eliminated (corrected).

The maximum forecast errors are presented in Table 14 .

Table 14: Maximum forecast errors

\begin{tabular}{|c|c|c|c|c|}
\hline Nr. crt & Distribution branch & ANN & CF & MLR \\
\hline 1 & Entire network assembly & 4.94 & 17.30 & 7.90 \\
\hline 2 & Distribution branch TM & 5.11 & 21.50 & 8.01 \\
\hline 3 & Distribution branch AR & 8.78 & 18.23 & 12.88 \\
\hline 4 & Distribution branch HD & 8.11 & 15.63 & 12.28 \\
\hline 5 & Distribution branch CS & 14.91 & 45.26 & 33.33 \\
\hline
\end{tabular}

The provided conclusions, regarding the quality of the results are sustained. The ones provided by the ANNs are the most acceptable ones. Among the conventional methods, the MLR based ones are the most suitable.

\section{Conclusion}

Accurate load forecasts are critical for distribution planning, for utilities. The quality of the forecast methods depends on the available historical data as well as on the knowledge about the main influence parameters on the energy consumption. 
Different forecasting methodologies have been integrated into a stand-alone application with a graphical user interface. The authors have applied these methodologies to obtain hourly load forecasts (for next 24 hours). A good performance and reasonable prediction accuracy was achieved for NN model.

The historical data preprocessing could be improved in order to obtain better results.

The developed software tool deals with real data; it leads to accurate consumed power forecasts. The conclusion is sustained through comparison performed with real monitored data for the same period.

The results are practically confirming the performed comments corresponding to the 20042013, respectively 2014-2016 periods.

Classical forecasting methods are not recommended to be applied. But, in case of MLR method a slight advantage is highlighted. Smallest forecasting errors have been obtained in case of ANN based method.

\section{Bibliography}

[1] Charytoniuk, W.; Chen, M.S.; Van Olinda, P. (1998). Nonparametric Regression Based Short-Team Load Forecasting, IEEE Transaction on Power Systems, 13(3), 735-730, 1998.

[2] Chen, H.; Canizares, A.C.; Ajit, S. (2011). ANN based Short-Term Load Forecasting in Electricity markets, Proceedings of the IEEE Power Engineering Society Winter Meeting, 2, 411-415, 2011.

[3] Chen, J.F.; Wang, W.M.; Huang, C.M.(2005). Analysis of an adaptive time-series autoregressive moving-average (ARMA) model for short-term load forecasting, Electric Power Systems Research, 34, 187-196, 2005.

[4] Chis, V.; Barbulescu, C., Kilyeni, S.; Dzitac S. (2018). Short-Term Load Forecasting Software Tool, Proceedings of the 7th International Conference on Computers Communications and Control (ICCCC), 111-118, 2018.

[5] Cho, M.Y.; Hwang, J.C.; Chen, C.S. (1995). Customer short-term load forecasting by using ARIMA transfer function model, Proceedings of the International Conference on Energy Management and Power Delivery, 317-322, 1995.

[6] Danladi, A.; Yohanna, M.; Puwu, M.I.; Garkida, B.M. (2016). Long-term load forecast modelling using a fuzzy logic approach, Pacific Science Review A: Natural Science and Engineering, 18(2), 123-127, 2016.

[7] Ho, K.l.; Hsu, Y.I.; Chen, C.F.; Lee, T.E.; Liang, C.C.; Lai, T.S.; Chen, K.K. (1990). Short Term Load Forecasting of Taiwan Power System Using a Knowledge Based Expert System, IEEE Transactions on Power Systems, 5(4), 1214-1221, 1990.

[8] Hong, W.C.; Dong, Y.; Chen, L.Y.; Wei, S.Y. (2012). Seasonal Support vector Regression with Chaotic Genetic Algorithm in Electric Load, ICGEC 6th International Conference on Genetic and Evolutionary Computing, 124-127, 2012.

[9] Hyndman, R.J.; Koehler, A.B. (2016). Another look at measuring forecast accuracy, International Journal of Forecasting, 22(2), 679-688, 2016.

[10] Ismail, Z.; Efendy, R. (2011). Enrollment forecasting based on modified weight fuzzy time series, Journal of Artificial Intelligence, 4(1), 110-118, 2011. 
[11] Jin, X.; Dong, Y.; Wu, J.; Wang, J. (2010). An Improved Combined Forecasting Method for Electric Power Load Based on Autoregressive Integrated Moving Average Model, International Conference of Information Science and Management Engineering (ISME), 2, 476-480, 2010.

[12] Karapidakis, S. (2007). Machine learning for frequency estimation of power systems, Applied Soft Computing, 7(1), 105-114, 2007.

[13] Mordjaoui, M.; Haddad, S.; Medoued, A.; Laouafi, A. (2017). Electric load forecasting by using dynamic neural network, Journal hydrogen Energy, 42, 17655-17663, 2017.

[14] Pandian, S.C.; Duraiswamy, K.; Rajan, C.C.A. (2006). Fuzzy approach for short term load forcasting, Electric Power Systems Research, 76, 541-548, 2006.

[15] Park, D.C.; El-Sharkawi, M.A.; Marks, R.J.; Atlas, L.E.; Damborg, M.J. (1991). Electric load forecasting using an artificial neural network, IEEE Transactions on Power Systems, 6(2), 442-449, 1991.

[16] Schellong, W. (2011). Energy Demand Analysis and Forecast, Energy Management Systems P. Giridhar Kini, IntechOpen, DOI: 10.5772/21022, 2011.

[17] Sheikh, S.K.; Unde, M.G. (2012). Short-Term Load Forecasting Using ANN Technique, International Journal of Engineering Sciences \& Emerging Technologies, 1(2), 97-107, 2012.

[18] Shelke, M.; Thakare, P.D. (2014). Short Term Load Forecasting by Using Data Mining Techniques, International Journal of Science and Research (IJSR), 3(9), 1363-1367, 2014.

[19] Singh, P.; Dwivedi, P. (2018). Integration of new evolutionary approach with artificial neural network for solving short term load forecast problem, Journal of Applied Energy, 217, 537$549,2018$.

[20] Srinivasan, D.S.; Tan, S.S.; Cheng, C.S.; Chan, E.K. (1999). Parallel neural network-fuzzy expert system strategy for short-term load forecasting: system implementation and performance evaluation, IEEE Transactions on Power Systems, 14(3), 1100-1106, 1999.

[21] Yang, H.P.; Yan, F.F.; Wang, H.; Zhang, L.(2016). Short-term load forecasting based on data mining, IEEE 20th International Conference on Computer Supported Cooperative Work in Design, 170-173, 2016.

[22] Zhang, J.,; Yi-Ming, W.; Dezhi, L.; Zhongfu, T.; Jianhua, Z. (2018). Short term electricity load forecasting using a hybrid model, Journal Energy, 158(C), 774-781, 2018.

[23] [Online]. Available: www.mathworks.com Matlab Users guide, Accesed on 12 February 2018. 